\title{
Arms Race Coevolution: The Local and Geographical Structure of a Host-Parasite Interaction
}

\author{
Rodrigo Medel • Marco A. Mendez • Carmen G. Ossa • \\ Carezza Botto-Mahan
}

Published online: 19 December 2009

(C) Springer Science+Business Media, LLC 2009

\begin{abstract}
Consideration of complex geographic patterns of reciprocal adaptation has provided insight into new features of the coevolutionary process. In this paper, we provide ecological, historical, and geographical evidence for coevolution under complex temporal and spatial scenarios that include intermittent selection, species turnover across localities, and a range of trait match/mismatch across populations. Our study focuses on a plant host-parasitic plant interaction endemic to arid and semiarid regions of Chile. The long spines of Chilean cacti have been suggested to evolve under parasite-mediated selection as a first line of defense against the mistletoe Tristerix aphyllus. The mistletoe, in turn, has evolved an extremely long morphological structure that emerges from the seed endosperm (radicle) to reach the host cuticle. When spine length was traced along cactus phylogenies, a significant association between spine length and parasitism was detected, indicating that defensive traits evolved in high correspondence with the presence or absence of parasitism in two cactus lineages. Assessment of spine-radicle matching across populations revealed a potential for coevolution in $50 \%$ of interaction pairs. Interestingly, hot spots for coevolution did not distribute at random across sites. On the contrary, interaction pairs showing high matching values occur mostly in the northern distribution of the interaction, suggesting a geographical structure for coevolution in this system. Only three sampled interaction pairs were so mismatched that reciprocal selection could not occur given current trait distributions. Overall, different lines of evi-
\end{abstract}

R. Medel $(\bowtie) \cdot$ M. A. Mendez $\cdot$ C. G. Ossa $\cdot$ C. Botto-Mahan Departamento de Ciencias Ecológicas, Facultad de Ciencias, Universidad de Chile,

Casilla 653,

Santiago, Chile

e-mail: rmedel@uchile.cl dence indicate that arms-race coevolution is an ongoing phenomenon that occurs in the global system of interconnected populations.

Keywords Geographic mosaic of coevolution - Adaptation . Host-parasite $\cdot$ Mistletoe $\cdot$ Cactus $\cdot$ Chile $\cdot$ Natural selection

\section{Introduction}

Host-parasite relationships have long attracted the attention of ecologists and evolutionary biologists because reciprocal adaptive responses may coevolve as a result of the antagonistic interaction. Implicit in most models of host-parasite coevolution is the idea that host characteristics providing defense against parasitism are adaptive and evolve under parasite-mediated selection, and infection parasite traits, in turn, evolve as a response to the host defensive traits evolved by host populations. In spite of its apparent simplicity, this idea has been difficult to evaluate empirically, and coadaptation of host and parasite traits is usually assumed rather than demonstrated. One possible reason for this is that reciprocal selection is not an all-ornone phenomenon that necessarily occurs in the overall range of localities where host and parasite coexist. This somewhat restricted and local view of coevolution has been recently expanded to a geographical perspective that takes into account a broader range of scenarios than previously considered. For example, at any point in space and time, interacting populations may occupy any position within the range of the coevolutionary race depending on the temperature of populations: from the early stages of escalation with a high rate of reciprocal selection (hotspots), to low reciprocal selection and cost-induced de-escalation (coldspots). Because a variety of historical, 
geographical, and ecological factors may influence the coevolutionary process, it is exceedingly difficult to draw inferences about the coevolutionary dynamics of interspecific interactions through the examination of one or a few localities at a single time. This perspective has been articulated into what has been called the Geographical Mosaic of Coevolution Theory (Thompson 1994, 2005), which takes explicitly into account the inherent complexity of local communities, the ample temporal and spatial variation in species composition and interactions, and the heterogeneity in the magnitude, direction, and shape of reciprocal selection across localities.

Arms race is a specific form of coevolution that is characterized by escalating levels of defense and counterdefense in antagonistic interactions. However, arms-race coevolution does not necessarily imply an endless increase in defense and counterdefense phenotypes. For example, the geographic structure of the interaction may prevent the relentless escalation through the acquisition of new defense mechanisms that may replace the original one in some populations (e.g., Benkman 1999; Benkman et al. 2003). Gene flow across localities may arm host species with a battery of possibilities including the original defense, the new defense, or a combination of both, probably depending on the level of overlapping between host and parasite populations (Nuismer et al. 2003) Similarly, gene flow between hotspots and coldspots will slow the rate of escalation in the global system of interconnected populations.

The arms race model for host-parasite interactions makes several specific predictions (Thompson 1994, 2005):

1. Local populations of the parasite are adapted to the least defended of their potential local host assemblage.

2. Parasite hierarchies of infection vary geographically, indicating host alternation across localities.

3. Some uninfected hosts exhibit high levels of defense, providing correlative evidence for past adaptive or anachronic traits to the interaction that have not yet been lost.

4. Some host populations may show low levels of defense, indicating that (1) the species is new to the antagonistic interaction or (2) the species is a host that lost its defense as the parasite focused on an alternative host species.

5. Host populations may show variable defense levels across localities, indicating different levels of defense ratcheting in different community contexts.

In this paper, we will focus on a specific hostparasite relationship to illustrate some elements of the arms race model in the context of the Geographical Mosaic of Coevolution Theory. To this end, we will use three approaches. First, we will use ecological observa- tions and experiments to identify: (a) the species with potential to exhibit arms-race coevolution, (b) the phenotypic traits involved in reciprocal selection, that is, those traits with a clear functional value that influence the distribution of mortality or fecundity on each population, and (c) the temporal dynamics of selection acting on the relevant functional traits. Second, we will use a phylogenetic approach to determine: (a) the pattern of trait evolution on a broad temporal scale and (b) the extent to which trait evolution is associated with the presence or absence of the interacting antagonistic species. Third, we will evaluate the actual geographic mosaic by (a) assessing the degree of matching and mismatching of coevolved traits across localities and (b) examining a potential regional structure of trait matching/mismatching in a multispecific context. Together, these approaches to coevolutionary analysis can help us to understand the extent to which geographic selection mosaics have shaped trait distribution across landscapes in this model system.

\section{Natural History and the Phenotypic Interface}

The parasitic habit in plants is represented by more than 3,000 species distributed in 16 families (Kuijt 1969; Musselman and Press 1995). In total, parasitic plants represent about $1 \%$ of the total species of angiosperms, and Loranthaceae is the most diversified family with ca. 700 species distributed in 22 genera around the world (Molau 1995). The family Loranthaceae diversified in warm climates, probably in closed forests in the midCretaceous about 70 million years ago (Barlow 1983). The South American genus Tristerix consists of 11 mistletoe species distributed in the west margin of the continent.

Tristerix aphyllus is a peculiar mistletoe endemic to the arid and semiarid regions of Chile that, unlike all other species of Loranthaceae, has leaves reduced to minute scales (Kuijt 1969, 1988; Fig. 1a). The leafless condition of $T$. aphyllus has been hypothesized to represent a derived adaptation to its peculiar life history that, unlike all other species in the Loranthaceae, includes cacti as the only host species (Mauseth 1991). T. aphyllus currently parasitizes several columnar cacti of the genera Echinopsis (Trichocereus) and Eulychnia. Different lines of geological, climatic, and biogeographical evidence suggest the association Tristerix-cacti may have originated about five million years ago (Medel et al. 2002). Since then, Tristerix has probably spread by including an increasing number of cactus species as hosts, adopting its current distribution in strict dependence upon cactus distribution. In Chile, the current distribution of the host-parasite interaction extends from $27^{\circ} \mathrm{S}$ to $35^{\circ} \mathrm{S}$. 
Fig. 1 The infection cycle in the cactus-mistletoe system. The fruits of the holoparasitic mistletoe Tristerix aphyllus (a) is one of the few feeding resources for the Chilean mockingbird, Mimus thenca (b). After eating the fruits, the bird often perches on the top of cacti and defecates the sticky mistletoe seeds that adhere to the surface or lateral spines of the new host (c). After some days, the seed has elongated a long radicle, (c) that eventually contacts the cactus epidermis to form a haustorial disk (d). The mistletoe cells enter to the cactus tissue, tap the phloematic vessels, and the parasitic plant grows inside for 18 months, after which the reproductive portion of the parasite emerges from the cactus surface (e) to repeat the cycle (f)
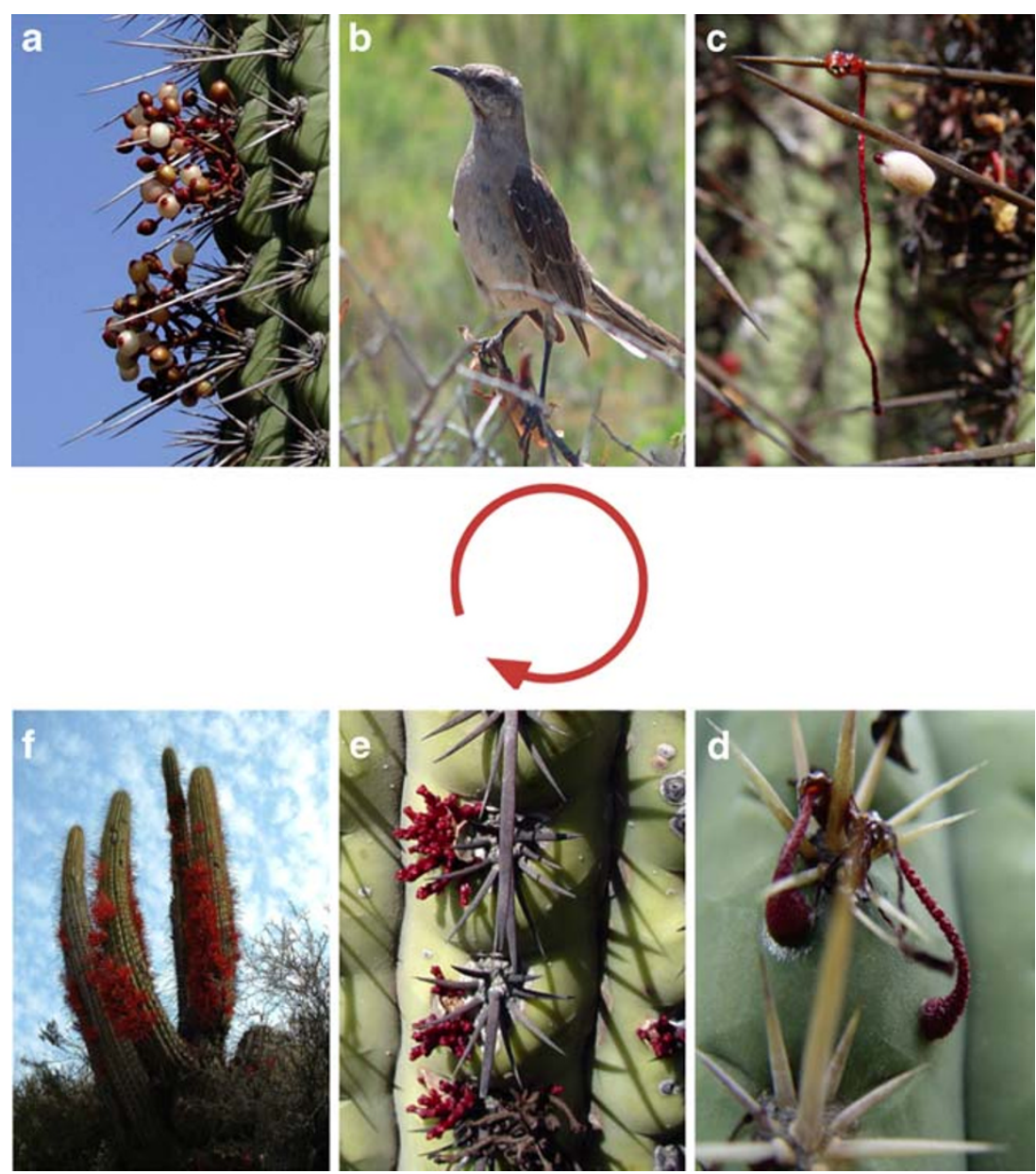

The infection by T. aphyllus spreads from one host to another through the Chilean mockingbird Mimus thenca (Mimidae), the only bird species responsible for disseminating the seeds of the mistletoe (Fig. 1b). The bird swallows whole ripe fruits and defecates the mucilaginous seeds intact (Martinez del Río et al. 1995). Seed deposition upon cacti is often aggregated and occurs especially on short-spined and previously parasitized individuals (Medel et al. 2004). Once defecated by the bird, the seeds often adhere to the spines of cacti and elongate a reddish morphological structure that protrudes from the seed endosperm (radicle, hereafter) that grows up to eight weeks (Fig. 1c) or until making contact with the epidermis of the cactus to form a morphological zone of contact (Fig. 1d) from which several filaments penetrate into cactus tissues through stomatal openings (see morphological details in Mauseth et al. 1984, 1985). Once inside the cactus, the plant grows for 18 months before emerging from the cactus surface as a red inflorescence to repeat the cycle (BottoMahan et al. 2000; Fig. 1e).

The spines of Chilean columnar cacti have a dual functional value in relation to parasitism by $T$. aphyllus. On the one hand, they represent a first barrier against infection by discouraging birds from perching on the top of the cactus columns. We have examined the role of cactus spines as a defensive trait against infection by T. aphyllus in a local system of two cactus species, Echinopsis chilensis and Eulychnia acida. Our results indicate that the visits performed by the bird vector to cacti and the mistletoe seed deposition tend to be low in long-spined individuals (Fig. 2). Spines of unvisited cacti E. chilensis and E. acida
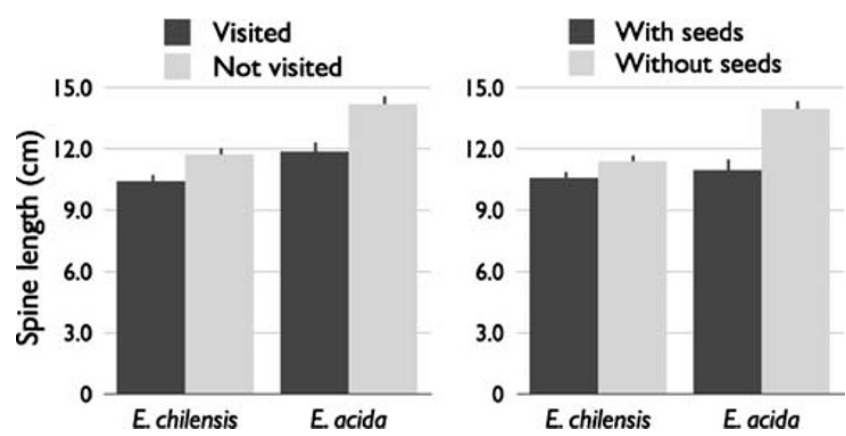

Fig. 2 Effect of spine length as a barrier to infection in Echinopsis chilensis and Eulychnia acida. Columns indicate mean (SE). Degrees of freedom are: E. chilensis $=1,217$ and $E$. acida $=1,66$. ${ }^{*} * P<0.01$, $* * * P<0.001$. See details in Medel 2000 
were 1.29 and $2.32 \mathrm{~cm}$ longer on average than spines of the visited individuals. Similarly, the spines of $E$. chilensis and E. acida without seed deposition were 0.84 and $3.05 \mathrm{~cm}$ longer on average, respectively, than spines of individuals with deposited seeds (Fig. 2). On the other hand, long spines prevent cacti from becoming infected when the first barrier is surpassed. By increasing the distance between the trapped sticky seeds and the cactus cuticle, they provide a physical barrier that prevents infection most of the time, excepting by mistletoe seeds with the ability to elongate extremely long radicles during the infection process. In this way, the ability of the parasites to reach the host and invade the cactus tissues is entirely dependent upon radicle length. Previous studies have shown a wide variation in radicle length in T. aphyllus (range: $40-90 \mathrm{~mm}$ after 40 days from bird defecation, Martinez del Río et al. 1995), reaching a maximum length of $137 \mathrm{~mm}$ (Medel et al. 2002). Interestingly, T. aphyllus has the longest radicle reported for species of the family Loranthaceae (Kuijt 1969). Assessment of association between radicle length and infection success revealed that most successful mistletoes had long radicles, indicating that spines of cacti prevent seeds with short-radicles from reaching the host cuticle (Gonzáles et al. 2007), hence favoring individuals with long radicles in the mistletoe population.

Because infection by T. aphyllus decreases fruit and seed production and often suppresses entirely cactus reproduction (Silva and Martinez del Río 1996; Medel 2000), shortspined individuals are strongly selected against, and long spines are expected to evolve as a result of parasitemediated selection. This expectation has been corroborated in a ten-year study of phenotypic selection on spine characters. Parasite-mediated selection, albeit variable across years, promotes long spines in E. chilensis and E. acida (Fig. 3), therefore satisfying one of the requirements for coevolution in this system (Medel 2000).

\section{The Phylogenetic Structure of the Coevolving System}

T. aphyllus currently parasitizes some but not all Echinopsis and Eulychnia species in Chile. For instance, several columnar cactus species currently inhabit places outside the geographical range of the parasitic plant, which suggests they have not had a history of association with the mistletoe. This geographical setting provides a useful scenario in which to evaluate the historical association of spine length with parasitism and to perform tests for correlated evolution in a phylogenetic context. Because phylogenies permit us to track the distribution of traits from a large-scale perspective, it is possible to understand the pathways followed by the coevolving interaction to its current geographical configuration.

\section{Echinopsis chilensis}

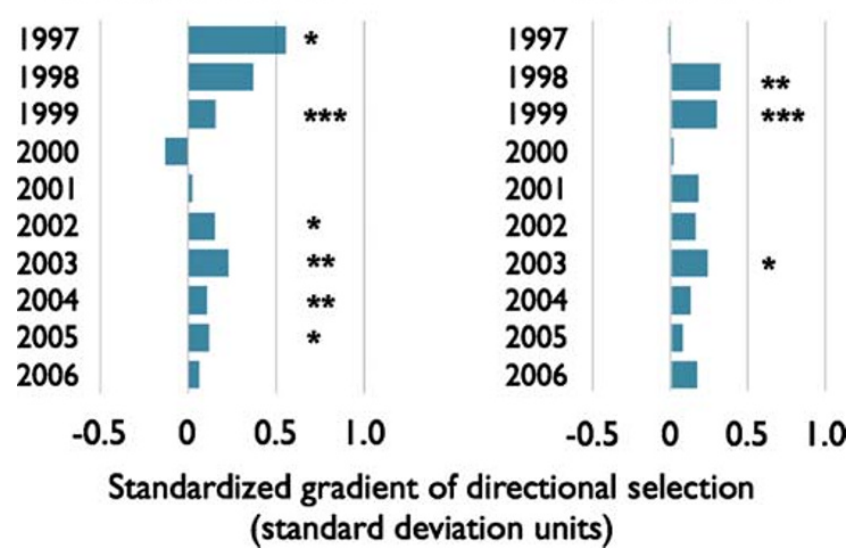

Fig. 3 Temporal pattern of directional selection $\left(\beta^{\prime}\right)$ on the spine length of the columnar cactus Echinopsis chilensis $(N=2,541)$ and Eulychnia acida $(N=459)$ during ten consecutive years at Las Chinchillas National Reserve. Cactus height and number of columns were considered as covariate in multivariate selection analyses (Lande and Arnold 1983; Brodie et al. 1995). Fruit production per cactus was considered as female fitness. Bars to the right of zero indicate selection in that year for plants with longer spines and bars to the left of zero indicate selection in that year for shorter spines. Asterisks indicate values significantly larger or smaller than zero. ${ }^{*} P<0.05$, $* * P<0.01, * * * P<0.001$

We collected samples for molecular analyses, recorded spine length, and measured the average parasite prevalence in a range of populations per host species. To investigate the extent to which transition in spine length along cactus phylogeny was associated with parasitism, we performed tests for correlated evolution between spine length and parasitism by assessing the fit of data to a model of random trait evolution (no correlation between variables) and to a model of correlated trait evolution. The model for correlated change provided a better fit than the model of independent change in the two cactus genera, indicating that transitions in spine length were dependent on parasitism in the two host phylogenies. Interestingly, the phylogenetic correlation between spine length and parasite prevalence fits well with the observation that the distribution range of short-spined cacti (Echinopsis deserticola, Echinopsis spinibarbis, Eulychnia iquiquensis, and Eulychnia saintpieana) does not overlap with $T$. aphyllus at present (Fig. 4). Notwithstanding, the observation that Echinopsis litoralis and Eulychnia castanea present short spines in spite of experiencing some level of infection suggests that these species have been only recently integrated into the coevolving system or have developed an alternative defensive mechanism.

In conclusion, phylogenetic evidence indicates that (1) host species that present high levels of infection at present tend to show long spines, (2) the host species living outside the distributional range of the mistletoe at present have 


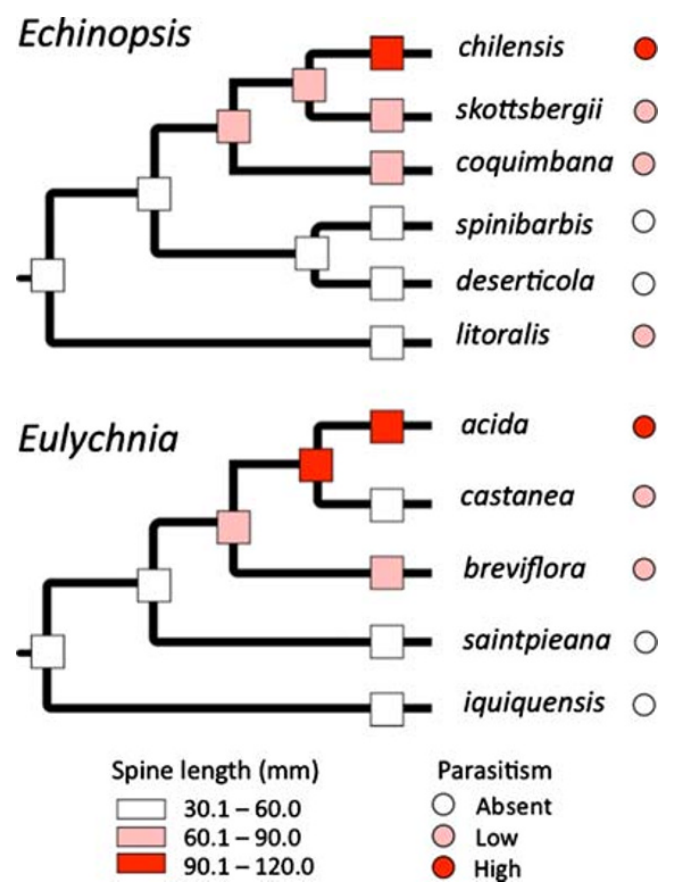

Fig. 4 Association between spine length and parasite infection in Chilean species of Echinopsis and Eulychnia. Spine length is depicted as squares over branches in the phylogeny, and the associated parasite prevalence is depicted in circles. Values of prevalence represent mean values in a range of 2-10 populations per cactus species. We used Corryocactus brevistylus, Browningia hertlingiana, and Oreocereus leucotrichus for outgroup-rooting in molecular phylogenetic reconstructions

shorter spines than their relatives probably because, unlike parasitized species, they have never been involved in armsrace coevolution, and (3) the pattern is consistent in host species belonging to different genera. All this evidence provides support to the idea that host-parasite coevolution occurs in a changing multispecific context across localities, therefore verifying a critical premise of the Geographic Mosaic of Coevolution Theory.

\section{Matches and Mismatches Across Populations}

Because the process of coevolution occurs on a wide spatial and temporal stage, we might observe a given pair of interacting populations occupying any position within the range of their coevolutionary trajectory. Analyses of the phenotypic interface of coevolution, defined as the set of traits that mediate interactions, help to understand reciprocal selection and therefore allow identification of hotspots and coldspots across the landscape (Brodie and Ridenhour 2003). Despite some variation in method and interpretation (e.g., Gomulkiewicz et al. 2007; Hanifin et al. 2008), matching and mismatching in the phenotypic interface may roughly represent the potential for reciprocal selection. High levels of phenotype matching imply a potential for strong reciprocal selection because any change in the phenotypic mean of one species is expected to have an important fitness impact on the other. Localities showing high levels of matching are referred to as hotspots to indicate a high potential for coevolution. High levels of mismatching, on the contrary, indicate that any change in one species will not necessarily have a fitness impact on the second interacting species, therefore reducing the chance of adaptive change by the second species. Localities showing a high level of mismatch are therefore referred as coldspots to denote a low potential for coevolution. In this particular arms-race coevolutionary model, the relevant phenotypic interface consists of traits that increase the chance of infection (i.e., radicle length) and prevent or reduce the chance of infection (i.e., spine length). Figure 5 depicts the geographical distribution of matches/mismatches in the host-parasite phenotypic interface. We detected roughly matched phenotypes and hence potential for coevolution in $50 \%$ (15 out of 30 ) of interaction pairs. Only four sampled interaction pairs showed extreme matching levels indicative of a strong potential for coevolution, and three localities
Fig. 5 Geographic distribution of hot, warm/hot, warm/cold, and cold spots for coevolution as revealed by the phenotypic interface between a host defensive trait (spine length) and a parasite infection trait (radicle length). Matching was recorded from $M=\mid$ spine length-radicle length $\mid$ : spine length. $M=1$ indicates perfect matching and high potential for coevolution, $\mathrm{M}=0$ indicates complete mismatch and minimum potential for coevolution




were so mismatched that reciprocal selection could not occur given current trait distributions. Interestingly, hotspots for coevolution did not distribute at random across sites, as they occurred mostly in the northern distribution of the interaction. This observation suggests a geographical structure for coevolution in this multispecific interaction system.

\section{Concluding Remarks}

We have presented different lines of evidence for arms-race coevolution in the mistletoe-cactus system. Identification of the relevant traits for the interaction has permitted an exploration of a range of questions that include local, phylogenetic, and geographical perspectives. Taken together, all the evidence indicates a phenomenon far more complex than previously thought. Intermittent local selection combined with a high host species turnover across localities indicates a very dynamic coevolutionary process that is reflected in the variable levels of host-parasite matching and mismatching at the regional scale. In spite of this community complexity, however, the long-term phylogenetic signal indicates a strong association between host defensive characters and parasitism, indicating that the mistletoe is responsible for the extremely long spines presented by some Chilean columnar cacti, and cacti have promoted the extremely long radicle in the mistletoe. Overall, these evidences indicate that regardless of the inherent community complexity at each locality, arms-race coevolution is an ongoing process in the global system. Current work on the phylogeographic structure of the interaction will help us to understand the genetic and historical determinants of the cactus-mistletoe coevolving system across landscapes.

Acknowledgments We thank Paula Caballero, Mildred Ehrenfeld, Wilfredo Gonzáles, Carlos Martinez del Río, Talía del Pozo, Eric Rivera, Arturo Silva, Eliseo Vergara, and the family Montero Peña for their collaboration in different steps of this research. CONAF IV Region and the personnel of Las Chinchillas National Reserve provided invaluable support during this research. John Thompson and Niles Eldredge made important comments that improved the clarity of this manuscript. This work was funded by grants FONDECYT 1970497, 1010660, PSD 66, and ACT 34/2006 to RM.

\section{References}

Barlow BA. Biogeography of Loranthaceae and Viscaceae. In: Calder M, Bernhardt P, editors. The biology of mistletoes. Sydney: Academic; 1983. p. 19-46.

Benkman CW. The selection mosaic and diversifying coevolution between crossbills and lodgepole pine. Am Nat. 1999;153:S75-91.
Benkman CW, Parchman TL, Favis A, Siepielski AM. Reciprocal selection causes a coevolutionary arms race between crossbills and lodgepole pine. Am Nat. 2003;162:182-94.

Botto-Mahan C, Medel R, Ginocchio R, Montenegro G. Factors affecting the circular distribution of the leafless mistletoe Tristerix aphyllus (Loranthaceae) on the cactus Echinopsis chilensis. Rev Chil Hist Nat. 2000;73:525-31.

Brodie ED III, Ridenhour BJ. Reciprocal selection at the phenotypic interface of coevolution. Integr Comp Biol. 2003;43:408-18.

Brodie ED III, Moore AJ, Janzen FJ. Visualizing and quantifying natural selection. Trends Ecol Evol. 1995;10:313-8.

Gomulkiewicz R, Drown DM, Dybdahl MF, Godsoe W, Nuismer SL, Pepin KM, et al. Dos and don'ts of testing the geographic mosaic theory of coevolution. Heredity. 2007;98:249-58.

Gonzáles WL, Suarez LH, Medel R. Phenotypic plasticity in the holoparasitic mistletoe Tristerix aphyllus (Loranthaceae): consequences of trait variation for successful establishment. Evol Ecol. 2007;21:431-44.

Hanifin CT, Brodie ED Jr, Brodie ED III. Phenotypic mismatches reveal escape from arms-race coevolution. Plos Biol. 2008;6:471-82.

Kuijt J. The biology of parasitic flowering plants. Berkeley: University of California Press; 1969. p. 246.

Kuijt J. Revision of Tristerix (Loranthaceae). Syst Bot Monogr. 1988;19:1-61.

Lande R, Arnold SJ. The measurement of selection on correlated characters. Evolution. 1983;37:1210-26.

Martinez del Río C, Hourdequin M, Silva A, Medel R. The influence of cactus size and previous infection on bird deposition of mistletoe seeds. Aust J Ecol. 1995;20:571-6.

Mauseth JD. Botany: an introduction to plant biology. Philadelphia: Saunders College Publishing; 1991.

Mauseth JD, Montenegro G, Walckowiak AM. Studies of the holoparasite Tristerix aphyllus (Loranthaceae) infecting Trichocereus chilensis (Cactaceae). Can J Bot. 1984;62:847-57.

Mauseth JD, Montenegro G, Walckowiak AM. Host infection and flower formation by the parasite Tristerix aphyllus (Loranthaceae). Can J Bot. 1985;63:567-81.

Medel R. Assessment of parasite-mediated selection in a host-parasite system in plants. Ecology. 2000;81:1554-64.

Medel R, Vergara E, Silva A, Kalin-Arroyo M. Effects of vector behavior and host resistance on mistletoe aggregation. Ecology. 2004;85:120-6.

Medel R, Botto-Mahan C, Smith-Ramirez C, Méndez MA, Ossa CG, Caputo L, et al. Quantitative natural history of a host-parasite relationship: the Tristerix-cactus system in semiarid Chile. Rev Chil Hist Nat. 2002;75:127-40.

Molau U. Reproductive ecology and biology. In: Press MC, Graves JD, editors. Parasitic plants. London: Chapman and Hall; 1995. p. 141-76.

Musselman LJ, Press MC. Introduction to parasitic plants. In: Press MC, Graves JD, editors. parasitic plants. London: Chapman and Hall; 1995. p. 1-13.

Nuismer SL, Thompson JN, Gomulkiewicz R. Coevolution between hosts and parasites with partially overlapping geographic ranges. J Evol Biol. 2003;16:1337-45.

Silva A, Martinez del Río C. Effects of the mistletoe Tristerix aphyllus (Loranthaceae) on the reproduction of its cactus host Echinopsis chilensis. Oikos. 1996;75:437-42.

Thompson JN. The coevolutionary process. Chicago: University of Chicago Press; 1994. p. 376.

Thompson JN. The geographic mosaic of coevolution. Chicago: University of Chicago Press; 2005. p. 443. 